\title{
ON A FINITE DIMENSIONAL QUASI-SIMPLE MODULE
}

\author{
KWANGIL KOH AND JIANG LUH
}

1. If $R$ is a ring and $M$ is a (right) $R$-module such that $M R \neq 0$, then $M$ is said to be quasi-simple provided that

(Q1) the endomorphism (module endomorphism) ring of the quasiinjective hull of $M$ is a division ring,

(Q2) if $N$ is a nonzero submodule of $M$ then there is a nonzero endomorphism $f$ of $M$ such that $f(M) \subseteq N$.

Any simple module is clearly quasi-simple, however a quasi-simple module is not necessarily simple. For example, if $R$ is a semiprime ring with a uniform right ideal $U$ such that the right singular ideal of $R$ is zero then the regular $R$-module $U$ is quasi-simple since the endomorphism ring of its quasi-injective hull is a division ring $[5,1.7$, p. 263] and for any nonzero submodule $N$ of $U$ there is $x \in N$ such that $0 \neq x U \subseteq N$. Let us say that a quasi-simple module is finite dimensional if its quasi-injective hull is a finite dimensional vector space over its endomorphism ring. The main results in this paper are the following: Let $M$ be a faithful quasi-simple $R$-module for some ring $R$ and let $\tilde{M}$ be the quasi-injective hull of $M$. Let $D$ $=\operatorname{Hom}_{R}(\tilde{M}, \tilde{M})$ and if $N$ is a nonzero submodule of $M$ then define $K(N)=\operatorname{Hom}_{R}(N, N)$. Then for any nonzero submodule $N$ of $M$, $K(N)$ is a right order in $D, \widetilde{M}=D N$ and if $\left\{m_{i}\right\}_{i=1}^{n}$ is a finite sequence of $D$-linearly independent elements in $\tilde{M}$ and if $\{y\}_{i=1}^{n}$ is a finite sequence in $M$, then there exist $r \in R, 0 \neq k \in \operatorname{Hom}_{R}(M, M)$ such that $0 \neq\left. k\right|_{N} \in K(N)$ and $m_{i} r=k y_{i}$ for all $1 \leqq i \leqq n$. If, in addition, the singular submodule of $M$ is zero then, for any large right ideal $B$ of $R$ one can choose $r \in B$ and $0 \neq k \in \operatorname{Hom}_{R}(M, M)$ such that $0 \neq\left. k\right|_{N}$ $\in K(N)$ and $m_{i} r=k y_{i}$ for all $1 \leqq i \leqq n$. In case the set $\left\{m_{1}, m_{2}, \cdots\right.$, $\left.m_{n}\right\}$ is a basis for $\tilde{M}$ then $r$ is a regular element. If $R$ is a right Goldie prime ring and $U$ is a uniform right ideal then $U$ is a finite dimensional quasi-simple module. Conversely, if $R$ has a faithful finite dimensional quasi-simple right module $M$ then $R$ is a right Goldie prime ring, and $M$ is isomorphic to a uniform right ideal of $R$. Hence our theory above provides a new proof for Theorem 10 of [3, p. 603]. Let $D_{n}$ be the $n \times n$ matrix ring representing $\operatorname{Hom}_{D}(\tilde{M}, \tilde{M})$ relative to the basis $\left\{m_{1}, m_{2}, \cdots, m_{n}\right\}$ and $K(N)_{n}$ be the $n \times n$ matrix ring over $K(N)$. Let $R^{*}$ be the subring of $D_{n}$ which represents $R$ as a ring of linear transformations on $\widetilde{M}$ over $D$ relative to the same basis.

Received by the editors April 10, 1969. 
Then $K(N)_{n} \cap R^{*}$ is a right order in $D_{n}$. We identify $D_{n}$ and $\operatorname{Hom}_{D}(\tilde{M}, \tilde{M})$. For each $d \in D$, let $\bar{d}$ be a matrix in $D_{n}$ such that $m_{i} \bar{d}=d m_{i}$ for all $1 \leqq i \leqq n$. Let $[K(N)]=\left\{\bar{d} \in D_{n} \mid d \in K(N)\right\}$. Then $[K(N)] \cap R^{*}$ is a right order in $\bar{D}$, where $\bar{D}=\left\{\bar{d} \in D_{n} \mid d \in D\right\}$. As one can see easily our theory here is a generalization of the density theorem for simple modules (refer to [4] and [7]).

2.0. Let $R$ be a ring and $M$ be an $R$-module (by an $R$-module we always mean a right $R$-module). We denote by $\hat{M}$ and $\tilde{M}$ respectively the injective hull and the quasi-injective hull of $M$. If $S$ and $T$ are nonempty subsets of $M$ and $R$ respectively, we define $S^{r}=\{r \in R \mid S r$ $=0, T^{r}=\{r \in R \mid T r=0\}$, and $T^{l}=\{m \in M \mid m T=0\}$. If $S=\{s\}$ and $T=\{t\}$ are singleton sets, we let $(s)^{r}=S^{r},(t)^{r}=T^{r}$, and $(t)^{l}=T^{l}$. If $M$ is a quasi-simple $R$-module and $A$ and $B$ are ideals of $M$ such that $M A B=0$ then either $M A=0$ or $M B=0$. Otherwise, one can find a nonzero $f \in \operatorname{Hom}_{R}(M, M)$ such that $f(M) \subseteq M A$ and hence $f(M B)$ $=f(M) B \subseteq M A B=0$. Since the kernel of $f$ is zero this implies that $M B=0$ which is absurd. Therefore $M^{r}$ is a prime ideal. We also note here that any pair of nonzero submodules of $\tilde{M}$ has a nonzero intersection since $\operatorname{Hom}_{R}(\tilde{M}, \tilde{M})$ is a division ring.

2.1. Proposition. Let $M$ be an $R$-module for some ring $R$ and let $D=\operatorname{Hom}_{R}(\tilde{M}, \tilde{M})$. Then $\tilde{M}=D M$.

Proof. Clearly $D M \subseteq \tilde{M}$. Let $\Lambda=\operatorname{Hom}_{R}(\hat{M}, \hat{M})$. Then by $[2,1$, p. 23], $\tilde{M}=\Lambda M$. If $x \in \bar{M}$, then $x=\sum_{i=1}^{n} f_{i}\left(m_{i}\right)$ for some positive integer $n$ where $f_{i} \in \Lambda$ and $m_{i} \in M$ for all $1 \leqq i \leqq n$. Let $d_{i}=f_{i} \mid \tilde{M}$. Since $\Lambda \tilde{M}=\tilde{M}, d_{i} \in D$, so $x \in D M$. Therefore $\tilde{M} \subseteq D M$.

2.2. Proposition. Let $M$ be a quasi-simple $R$-module for a ring $R$. Then, for any nonzero submodule $N$ of $M, \tilde{N}=\tilde{M}=D N$ where $D=\operatorname{Hom}_{R}(\tilde{M}, \tilde{M})$.

Proof. Since $D$ is a division ring, $M$ is an essential extension of $N$. Therefore $\hat{N}=\hat{M}$. Let $\Lambda=\operatorname{Hom}_{R}(\hat{M}, \hat{M})$. Then $\Lambda N=\tilde{N}$ by $[2,1$, p. 23], and hence $D N \subseteq \tilde{N}$. By (Q2), there is a nonzero $d \in D$ such that $d(M) \subseteq N$. Hence $D d(M) \subseteq D N \subseteq \tilde{N}$. However $D d=D$, since $D$ is a division ring. Thus $\tilde{M}=\bar{D} M=\bar{D} d(M) \subseteq D N \subseteq \tilde{N}$. Since $\tilde{N} \subseteq \tilde{M}$ always, this proves that $\tilde{M}=\tilde{N}=D N$.

2.3. Proposition. Let $M$ be a quasi-simple $R$-module for some ring R. If $N$ is any nonzero submodule of $M$, then $\tilde{M}^{r}=N^{r}$.

Proof. By 2.2. $\tilde{M}=\tilde{N}=D N$, where $D=\operatorname{Hom}_{R}(\tilde{M}, \tilde{M})$ is a division ring. It follows immediately that $N^{r}=(D N)^{r}=\tilde{M}^{r}$. 
2.4. TheOREM. Let $M$ be a quasi-simple $R$-module for some ring $R$ and let $D=\operatorname{Hom}_{R}(\tilde{M}, \tilde{M})$. If $N$ is a nonzero submodule of $M$ and if $K(N)=\operatorname{Hom}_{R}(N, N)$, then $K(N)$ is a right order in $D$.

Proof. Let $0 \neq d \in D$ and let $N(d)=\{m \in M \mid d(m) \in N\}$. Then $N(d)$ is a nonzero submodule of $M$. Let $N_{1}=N \cap N(d)$. Since $N_{1} \neq 0$, there is $f \neq 0$ in $\operatorname{Hom}_{R}(M, M)$ such that $f(M) \subseteq N_{1}$ by (Q2). Hence $d f(M) \subseteq d\left(N_{1}\right) \subseteq N$ and $\left.d f\right|_{N} \in K(N)$. This proves that $K(N)$ is a right order in $D$.

2.5. Proposition. Let $M$ be a quasi-simple $R$-module for some ring $R$ and let $D=\operatorname{Hom}_{R}(\tilde{M}, \tilde{M})$. If $\left\{x_{i}\right\}_{i=1}^{n}$ is a finite subset of $\tilde{M}$ and $y \in \tilde{M}$, then $y$ is a D-linear combination of $\left\{x_{i}\right\}_{i=1}^{n}$ if and only if $\bigcap_{i=1}^{n}\left(x_{i}\right)^{r} \subseteq(y)^{r}$.

Proof. If $y$ is a $D$-linear combination of $x_{1}, \cdots, x_{n}$, then clearly $\bigcap_{i=1}^{n}\left(x_{i}\right)^{r} \subseteq(y)^{r}$. Now, by [5, 2.2, p. 263], $\left(D x_{1}+D x_{2}+\cdots+D x_{n}\right)^{r l}$ $=D x_{1}+D x_{2}+\cdots+D x_{n}$. If $\bigcap_{i=1}^{n}\left(x_{i}\right)^{r} \subseteq(y)^{r}$ then $\left(D x_{1}+D x_{2}+\cdots+D x_{n}\right)^{r}$ $=\bigcap_{i=1}^{n}\left(x_{i}\right)^{r} \subseteq(y)^{r} \quad$ and $\quad y \in(y)^{r l} \subseteq\left(D x_{1}+D x_{2}+\cdots+D x_{n}\right)^{r b}=D x_{1}$ $+D x_{2}+\cdots+D x_{n}$.

2.6. Theorem. Let $R$ be a ring and $M$ be a quasi-simple $R$-module. Let $D=\operatorname{Hom}_{R}(\tilde{M}, \tilde{M})$ and let $K(N)=\operatorname{Hom}_{R}(N, N)$ where $N$ is a nonzero submodule of $M$. If $\left\{x_{i}\right\}_{i=1}^{n}$ is a finite D-linearly independent subset of $\tilde{M}$ and $\left\{y_{i}\right\}_{i=1}^{n}$ is a sequence in $M$ then there is $r \in R$ and $0 \neq k \in \operatorname{Hom}_{R}(M, M)$ such that $\left.k\right|_{N} \in K(N)$ and $x_{i} r=k y_{i}$ for all $1 \leqq i \leqq n$.

Proof. Define $I_{j}=\bigcap_{i=1 ; i \neq 1}^{n}\left(x_{i}\right)^{r}$. The $x_{j} I_{j} \neq 0$ for any $1 \leqq j \leqq n$. For, if $x_{j} I_{j}=0$ for some $j$, then $x_{j} \in \sum_{i=1 ; i \neq j}^{n} D x_{i}$ by 2.5. and the set $\left\{x_{i}\right\}_{i=1}^{n}$ would not be linearly independent. Hence $x_{j} I_{j} \cap N$ is a nonzero submodule of $M$. By (Q2), there is a nonzero $f_{j} \in \operatorname{Hom}_{R}(M, M)$ such that $f_{j}(M) \subseteq x_{j} I_{j} \cap N$. Hence $f_{j}\left(y_{j}\right) \in x_{j} I_{j} \cap N$, and $0 \neq\left. f_{j}\right|_{N}$ $\in K_{R}(N)$. Thus, if $y_{j} \neq 0$, there exists $a_{j} \in I_{j}$ such that $x_{j} a_{j}=f_{j} y_{j} \neq 0$. Without loss of generality, we may assume that $y_{j} \neq 0$ for $1 \leqq j \leqq m$ and $y_{j}=0$ for $m<j \leqq n$. Then $\left(\bigcap_{j=1}^{m} f_{j} y_{j} R\right) \cap N$ is a nonzero submodule of $N$. Hence there is $0 \neq k_{0} \in \operatorname{Hom}_{R}(M, M)$ such that $k_{0}(M)$ $\subseteq\left(\bigcap_{j=1}^{m} f_{j} y_{j} R\right) \cap N$ and $k_{0} y_{j}=f_{j} y_{j} r_{j} \neq 0$ for some $r_{j} \in R, 1 \leqq j \leqq m$, also $k_{0 \mid N} \in K(N)$ since $k_{0}(N) \subseteq N$. Now we let $r=\sum_{i=1}^{m} a_{i} r_{i}$. Then $x_{i} r=k_{0} y_{i}$ for all $1 \leqq i \leqq n$.

2.7. Proposition. Let $M$ be a quasi-simple $R$-module for some ring $R$. If the singular submodule of $M$ is zero, then $M$ is a quasi-simple $B$ module for any large right ideal $B$ of $R$. 
Proof. Let $f \in \operatorname{Hom}_{B}(N, M)$ for a submodule $N$ of $M$. Suppose $f\left(m r_{0}\right)-f(m) r_{0} \neq 0$ for some $m \in N$ and $r_{0} \in R$. Let $A=\left\{a \in R \mid r_{0} a \in B\right\}$. Since $B$ is a large right ideal, so is $A$. Let $G=A \cap B$. Then $G$ is also a large right ideal of $R$ and $\left(f\left(m r_{0}\right)-f(m) r_{0}\right) g=f\left(m r_{0}\right) g-f(m) r_{0} g$ $=f\left(m r_{0} g\right)-f\left(m r_{0} g\right)=0$ for any $g \in G$. Since the singular submodule of $M$ is zero, this implies that $f\left(m r_{0}\right)-f(m) r_{0}=0$, a contradiction. Thus $f \in \operatorname{Hom}_{R}(N, M)$ and $\operatorname{Hom}_{R}(N, M) \subseteq \operatorname{Hom}_{B}(N, M) \subseteq \operatorname{Hom}_{R}(N, M)$. Therefore $\operatorname{Hom}_{R}(N, M)=\operatorname{Hom}_{B}(N, M)$. Likewise, since the singular submodule of $\tilde{M}$ is zero, $\operatorname{Hom}_{R}(\tilde{M}, \tilde{M})=\operatorname{Hom}_{B}(\tilde{M}, \tilde{M})$. Now if $N$ is a nonzero $B$-submodule of $M$, then $N B \subseteq N$ and $N B \neq 0$. Since $N B$ is a nonzero $R$-submodule of $M$, there is $0 \neq f \in \operatorname{Hom}_{R}(M, M)$ $=\operatorname{Hom}_{B}(M, M)$ such that $f(M) \subseteq N B \subseteq N$. This proves that $M$ is a quasi-simple $B$-module.

2.8. CoRollary. If the singular submodule of $M$ in 2.6. is zero then, for any large right ideal $B$ of $R$, one can choose $b \in B$ and $0 \neq k$ $\in \operatorname{Hom}_{R}(M, M)$ such that $\left.k\right|_{N} \in K(N)$ and $x_{i} b=k y_{i}$ for all $1 \leqq i \leqq n$.

Proof. This is a direct consequence of 2.6. and 2.7.

2.9. TheOREM. Let $R$ be a ring and $M$ be a faithful finite dimensional quasi-simple $R$-module. Then every large right ideal $B$ of $R$ contains a regular element.

Proof. Let $M_{\tau}^{\Delta}$ be the singular submodule of $M$. We will show that $M_{R}^{\Delta}=0$. Suppose that $M_{r}^{\Delta} \neq 0$, then by $2.2 \tilde{M}=D M_{r}^{\Delta}$ where $D$ $=\operatorname{Hom}_{R}(\tilde{M}, \tilde{M})$. Since $M$ is finite dimensional, there exists a finite basis $\left\{m_{1}, m_{2}, \cdots, m_{n}\right\}$ in $M_{r}^{\Delta}$ such that $\tilde{M}=\sum_{i=1}^{n} D m_{i}$. It would follow that $\tilde{M}^{r}=\bigcap_{i=1}^{n}\left(m_{i}\right)^{r} \neq 0$ contradicting the faithfulness of $M$. Now, let $\left\{x_{1}, x_{2}, \cdots, x_{n}\right\}$ be a basis for $\tilde{M}$, where $x_{i}$ can be chosen from $M$ by 2.2. Then by 2.8 , there are $b \in B$ and $0 \neq k \in \operatorname{Hom}_{R}(M, M)$ such that $x_{i} b=k x_{i}$ for all $1 \leqq i \leqq n$. Since $\left\{k x_{1}, k x_{2}, \cdots, k x_{n}\right\}$ is also a basis of $\tilde{M}, b$ must be a regular element.

3.0. Proposition. If $R$ is a prime right Goldie ring, then there is a faithful finite dimensional quasi-simple $R$-module $M$.

Proof. Take $M$ to be any uniform right ideal of $R$. Then the regular $R$-module $M$ is a faithful quasi-simple $R$-module as was proven in $\$ 1$. It is well known that if $m \in M$ then $(m)^{r}$ is a closed right ideal. Suppose that $M$ were not finite dimensional over $D$, where $D=\operatorname{Hom}_{R}(\tilde{M}, \tilde{M})$, and that $\left\{m_{1}, m_{2}, \cdots\right\}$ were an infinite $D$-independent set in $M$. Since any right Goldie ring satisfies the descending chain condition on closed right ideal (see for example [5, 3, p. 264]), the sequence $\left(m_{1}\right)^{r} \supset\left(m_{1}\right)^{r} \cap\left(m_{2}\right)^{r} \supset \cdots$, would terminate at finite 
number of places, say $\bigcap_{i=1}^{n}\left(m_{i}\right)^{r}=\bigcap_{i=1}^{n+1}\left(m_{i}\right)^{r}$. Thus, $\left(m_{n+1}\right)^{r} \supseteq \bigcap_{i=1}^{n}\left(m_{i}\right)^{r}$ and $m_{n+1} \in D m_{1}+D m_{2}+\cdots+D m_{n}$ by 2.5 , a contradiction. Therefore $M$ is finite dimensional.

If $R$ is a prime ring with zero singular ideal and if $R$ contains a uniform right ideal then the injective hull $\hat{R}$ of the regular $R$-module $R$ is a prime regular ring with a minimal right ideal (refer to $[6,2.7$ and 3.1]). Furthermore, if $a \in R$ such that $(a)^{r}=0$ then there is $x \in \hat{R}$ such that $x a=1$. We use these facts to prove the following theorem.

3.1. TheOREM. Let $R$ be a ring and $M$ be an n-dimensional faithful quasi-simple R-module for some positive integer n. Let $D=\operatorname{Hom}_{R}(\tilde{M}, \tilde{M})$ and $\&=\operatorname{Hom}_{D}(\tilde{M}, \tilde{M})$. Then the injective hull $\hat{R}$ of the regular $R$-module $R$ is isomorphic (ring) to 2 .

Proof. First we note that by $2.0, M^{r}=0$ is a prime ideal of $R$, so $R$ is a prime ring. According to 2.9, the singular ideal of $R$ is zero. Let $m$ be a nonzero element of $M$. Then there exists a nonzero right ideal $U$ in $R$ such that $(m)^{r} \cap U=0$. Since every pair of nonzero submodules of $M$ has a nonzero intersection, $U$ must be a uniform right ideal of $R$. Thus $\hat{R}$ is a prime regular ring with a minimal right ideal. Let $S$ be an arbitrary nonzero ideal in $\hat{R}$. Then $S \cap R$ is a nonzero ideal in $R$ and it is a large right ideal of $R$. Hence, by $2.9, S \cap R$ contains a regular element, say $a$. Therefore, there is $x \in \hat{R}$ such that $1=x a \in x S \subseteq S$. Thus $S=\hat{R}$. This means that $\hat{R}$ is a simple ring with a minimal right ideal. Since $1 \in \hat{R}, \hat{R}$ is a simple artinian ring, and is isomorphic to the ring of all linear transformations of a finite dimensional vector space. Any $a \in \hat{R}$ such that either $a x=1$ or $x a=1$ for some $x \in R$ is a unit. Hence in particular any regular element of $R$ is a unit in $\hat{R}$. Since if $q \in \hat{R}$ then the right ideal $(R: q)=\{r \in R \mid q r \in R\}$ is large, by 2.9 there is a regular element $a \in(R: q)$ such that $q a=b \in R$. Therefore $q=b a^{-1}$ and $R$ is a right order in $\hat{R}$. Hence $\hat{R}$ is isomorphic to a subring of $\mathscr{L}$ and $\tilde{M}$ is a faithful $\hat{R}$-module. Since every $\tilde{R}$-module is injective (see $[1,4.2$, p. 11]) and $\tilde{M}$ is a uniform $\hat{R}$-module, $\tilde{M}$ has no proper nonzero submodules. Note that $\operatorname{Hom}_{\hat{R}}(\tilde{M}, \tilde{M})=\operatorname{Hom}_{R}(\tilde{M}, \tilde{M})$. Thus $\tilde{M}$ is an $n$-dimensional $\hat{R}$-module and $R \cong \mathscr{L}$.

3.2. Theorem. A ring $R$ has a faithful finite dimensional quasisimple right module if and only if $R$ is a right Goldie prime ring. When the conditions hold, a faithful right $R$-module $M$ is finite dimensional and quasi-simple if and only if it is isomorphic to a uniform right ideal of $R$.

Proof. It is clear that every Goldie prime ring has a uniform right ideal which is a faithful finite dimensional quasi-simple right module. 
Now we assume that $R$ has a faithful finite dimensional quasi-simple right module, say $M$. Let $D=\operatorname{Hom}_{R}(\tilde{M}, \tilde{M})$ and $\mathscr{L}=\operatorname{Hom}_{D}(\tilde{M}, \tilde{M})$. By the proof of $3.1, \mathcal{L}$ is a simple artinian ring and $R$ is a right order in $\mathcal{L}$. Hence $R$ is a right Goldie prime ring. Moreover, since $\widetilde{M}$ has no proper nonzero submodule, $\tilde{M}_{\mathscr{L}}$ is isomorphic to a minimal right ideal of $\mathcal{L}$. Identify $\tilde{M}$ with that right ideal. Since $R$ is large in $\mathfrak{L}_{R}$, $M \cap R \neq 0$, and since $M \cap R \subseteq \tilde{M}$, we see that $M \cap R$ is a uniform right ideal of $R$. Finally, by the definition of quasi-simplicity, there is a nonzero $f \in D$ such that $f(M) \subseteq M \cap R$. Since all elements of $D$ are monomorphisms, the right ideal $f(M)$ is the required isomorphic copy of $M$.

3.3. Lemma. Let $D$ be a division ring and $K$ be a right order in $D$. Then the $n \times n$ matrix ring $K_{n}$ over $K$ is a right order in $D_{n}$ where $D_{n}$ is the $n \times n$ matrix ring over $D$ and, for any nonsingular matrix $\left(k_{i j}\right) \in K_{n}$, there exists a scalar matrix $\bar{k}$ which is determined by some $k \in K$ and $a$ matrix $\left(k_{i j}^{\prime}\right) \in K_{n}$ such that $\left(k_{i j}\right)^{-1}=\left(k \bar{k}_{i j}^{\prime}\right)^{-1}$.

Proof. See [8, p. 114].

3.4. Theorem. Let $R$ be a ring and $M$ be an n-dimensional faithful quasi-simple R-module for some positive integer n. Let $D=\operatorname{Hom}_{R}(\tilde{M}, \tilde{M})$ and $K(N)=\operatorname{Hom}_{R}(N, N)$, where $N$ is a nonzero submodule of $M$. Let $R^{*}$ be the subring of $D_{n}$ which represents $R$ as a ring of linear transformations on $\tilde{M}$ over $D$ relative to the basis on which $D_{n}$ represents $\operatorname{Hom}_{D}(\tilde{M}, \tilde{M})$. Then $K(N)_{n} \cap R^{*}$ is a right order in $D_{n}$ and $[K(N)] \cap R^{*}$ is a right order in $\bar{D}$ where $[K(N)], \bar{D}$ are the rings of scalar matrices over $K(N)$ and $D$ respectively.

Proof. By 2.2, $\tilde{M}=D N$. Let $\left\{m_{1}, m_{2}, \cdots, m_{n}\right\} \subseteq N$ be a basis of $\tilde{M}$ on which $D_{n}$ represents $\mathscr{L}=\operatorname{Hom}_{D}(\tilde{M}, \tilde{M})$. If $q \in D_{n}$ then $q=\left(k_{i j}\right) \bar{k}^{-1}$ for some $\left(k_{i j}\right) \in K(N)_{n}$ and $\bar{k} \in[K(N)]$ by 3.2. By 3.1, $q=r_{1} r_{2}^{-1}$ for some $r_{1}, r_{2} \in R^{*}$. Since $\bar{k}^{-1} r_{2}$ is regular, the set $\left\{m_{1} \bar{k}^{-1} r_{2}\right.$, $\left.m_{2} \bar{k}^{-1} r_{2}, \cdots, m_{n} \bar{k}^{-1} r_{2}\right\}$ is linearly independent. Hence by 2.6 there exist $r \in R^{*}$ and $0 \neq k_{0} \in \operatorname{Hom}_{R}(M, M)$ such that $m_{i} \bar{k}^{-1} r_{2} r=k_{0} m_{i}=m_{i} \bar{k}_{0}$ for all $1 \leqq i \leqq n$ and $k_{\left.0\right|_{N}} \in K(N)$. This means that $\bar{k}^{-1} r_{2} r=\bar{k}_{0}$ and $r_{2} r=\bar{k} \bar{k}_{0} \in[K(N)] \cap R^{*}$. Now $r_{1} r=\left(k_{i j}\right) \bar{k}^{-1} r_{2} r=\left(k_{i j}\right) \bar{k}_{0} \in K(N)_{n} \cap R^{*}$. Therefore $q=\left(k_{i j}\right) \bar{k}^{-1}=\left(k_{i j}\right) \bar{k}_{0} \bar{k}_{0}^{-1} \bar{k}^{-1}=\left(r_{1} r\right)\left(r_{2} r\right)^{-1}$ and $K(N)_{n} \cap R^{*}$ is a right order in $D_{n}$. To see that $[K(N)] \cap R^{*}$ is a right order in $\bar{D}$, let $\bar{d} \in \bar{D}$. Then $\bar{d}=\left(k_{i j}\right) \bar{k}^{-1}$ for some $\left(k_{i j}\right) \in K(N)_{n} \cap R^{*}$ and $\bar{k} \in[K(N)]$ $\cap R^{*}$ by the above argument. Therefore $\bar{d} \bar{k}=\left(k_{i j}\right)$. Now $m_{i} \bar{d} \bar{k}$ $=m_{i}\left(k_{i j}\right)$, for all $1 \leqq i \leqq n$, so

$$
\begin{aligned}
k_{i j} & =d k & & \text { if } i=j, \\
& =0 & & \text { otherwise. }
\end{aligned}
$$


This means that $\left(k_{i j}\right) \in[K(N)] \cap R^{*}$ and $[K(N)] \cap R^{*}$ is a right order in $\bar{D}$.

The authors wish to thank the referee for many helpful comments. In particular, Theorem 3.2 is suggested by the referee.

\section{REFERENCES}

1. H. Cartan and S. Eilenberg, Homological algebra, Princeton Univ. Press, Princeton, N. J., 1956. MR 17, 1040.

2. C. Faith, Lectures on injective modules and quotient rings, Lecture Notes in Math., no. 49, Springer-Verlag, Berlin and New York, 1967. MR 37 \#2791.

3. A. W. Goldie, The structure of prime rings under ascending chain conditions, Proc. London Math. Soc. (3) 8 (1958), 589-608. MR 21 \#1988.

4. N. Jacobson, Structure of rings, 2nd rev. ed., Amer. Math. Soc. Colloq. Publ., vol. 37, Amer. Math. Soc., Providence, R. I., 1964. MR 36 \#5158.

5. R. E. Johnson and E. T. Wong, Quasi-injective modules and irreducible rings, J. London Math. Soc. 36 (1961), 260-268. MR 24 \#A1295.

6. R. E. Johnson, Quotient rings of rings with zero singular ideals, Pacific J. Math. 11 (1961), 1385-1392. MR 26 \#1331.

7. K. Koh and A. C. Mewborn, The weak radical of a ring, Proc. Amer. Math. Soc. 18 (1967), 554-559. MR 35 \#4252.

8. J. Lambek, Lectures on rings and modules, Blaisdell, Waltham, Mass., 1966. MR 34 \#5857.

North Carolina State University, Raleigh, North Carolina 27607 